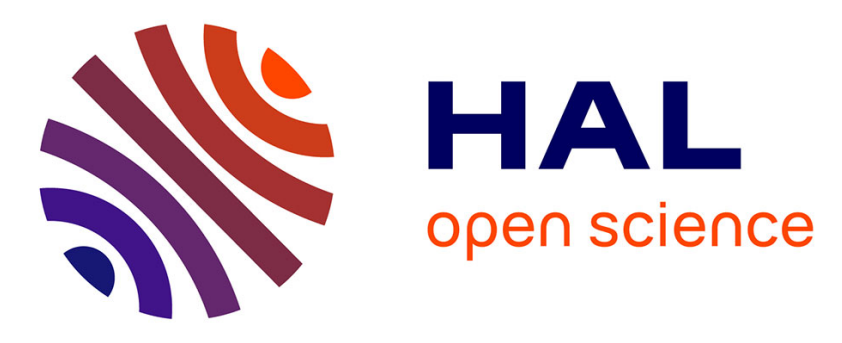

\title{
Chipless RFID Temperature and Humidity Sensing
}

\author{
Florian Requena, Nicolas Barbot, Darine Kaddour, Etienne Perret
}

\section{To cite this version:}

Florian Requena, Nicolas Barbot, Darine Kaddour, Etienne Perret. Chipless RFID Temperature and Humidity Sensing. 2021 IEEE/MTT-S International Microwave Symposium - IMS 2021, Jun 2021, Atlanta, United States. pp.545-548, 10.1109/ims19712.2021.9574942 . hal-03413126

\section{HAL Id: hal-03413126 \\ https://hal.science/hal-03413126}

Submitted on 5 Nov 2021

HAL is a multi-disciplinary open access archive for the deposit and dissemination of scientific research documents, whether they are published or not. The documents may come from teaching and research institutions in France or abroad, or from public or private research centers.
L'archive ouverte pluridisciplinaire HAL, est destinée au dépôt et à la diffusion de documents scientifiques de niveau recherche, publiés ou non, émanant des établissements d'enseignement et de recherche français ou étrangers, des laboratoires publics ou privés. 


\title{
Chipless RFID Temperature and Humidity Sensing
}

\author{
Florian Requena ${ }^{1}$, Nicolas Barbot, Darine Kaddour, Etienne Perret \\ Univ. Grenoble Alpes, Grenoble INP, LCIS, F-26000 Valence, France \\ ${ }^{1}$ florian.requena@lcis.grenoble-inp.fr
}

\begin{abstract}
In this paper, a temperature and humidity chipless RFID sensor is presented. A conventional chipless RFID interrogation process is used to extract the tag identification as well as the two physical parameters. A model describing the evolution of the resonance frequency of a scatterer as a function of temperature and humidity is introduced. It allows the two quantities to be extracted independently of each other while each of them impacts the resonance frequency. On average, an error lower than $0.7{ }^{\circ} \mathrm{C}$ is obtained on the temperature and less than $1.3 \%$ on the humidity.

Keywords - Chipless RFID, Scatterer, Temperature, Humidity, Wireless sensor.
\end{abstract}

\section{INTRODUCTION}

In today's state of the art, multiple chipless RFID sensors have been made [1], [2], [3] including temperature sensors [4], [5]. An important drawback of these works is that they only consider the thermal effect of the environment on the tag whereas in a real-life applications, humidity will also be present and impact the resonance frequency which is used to extract the temperature. This is mainly because most of these works do not use an analytical model but an empirical approach that does not allow to dissociate the influence of temperature from the humidity. These two quantities will together impact the resonance frequency which is the only data extracted for the sensor functionality in these works. Indeed, water molecules in the air can be absorbed by the substrate and increase its permittivity ( frequency of a few $\mathrm{GHz}$ ). However, since actual works only focus on the thermal impact on the resonance frequency, the real-life application of these sensors is arguable. In this paper, a method to isolate and sense at the same time both temperature and humidity is proposed.

\section{THEORY}

The measurement principle based on a radar approach is illustrated in Fig. 1. When a loop resonator is illuminated by an incoming electromagnetic-field (see Fig. 1), the maximum energy back-scattered at a temperature $T$ occurs at its resonant frequency $f$ defined by [6]:

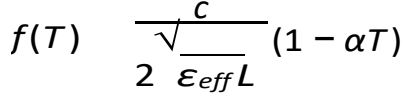

where $c$ is the speed of light, $L$ the effective length of the resonator, $\varepsilon_{\text {eff }}$ the effective permittivity seen by the resonator and $\alpha$ is defined by:

$$
\alpha=\alpha_{c}+\alpha_{d} / 2
$$

with $\alpha_{d}$ as the thermal-dependency on the effective permittivity and $\alpha_{c}$ as the coefficient of effective dilatation of the resonator. In the most general case, the substrate dependence for humidity is unknown but by considering small variations of humidity around a specific value $R H_{0}$, where $\mathrm{RH}$ denotes the relative humidity (in \%) seen by the resonator, it is possible to linearize the permittivity:

$$
\varepsilon(T, R H) \begin{array}{cc}
\varepsilon_{r}(R H)\left(\varepsilon_{r}\left(R^{+} \beta_{0}\right)\right. & \varepsilon_{r}\left(R H_{0}\right)(1+B T) \\
\times \quad 1+\frac{\varepsilon_{r}}{\varepsilon_{r}\left(R H_{0}\right)} & 0
\end{array}
$$

which can be noted as:

$$
\varepsilon(T, R H)=\varepsilon_{r}\left(R H_{0}\right)(1+B T)(1+[a+\eta R H]) .
$$

In this case, (1) can be extended to:

$$
f(T, R H) \quad \frac{{ }^{c}}{2 \bar{\varepsilon}_{e f f} L}\left(1-\alpha T-\left[a^{\prime}+\gamma R H\right]\right)
$$

where $\gamma$ is linked to $\eta$ and $a^{\prime}$ to $a$, the same way $b$ is linked to $\alpha_{d}$ [6] and $\varepsilon_{\text {eff }}$ is calculated using [7]. Notice that the thermal and humidity dependency on the permittivity is expressed by the coefficients $\alpha, a^{\prime}$ and $\gamma$ in (5). For this reason, the value of $\varepsilon_{\text {eff }}$ in (5) is not dependent on these parameters anymore.

If we have two resonators (noted 1 and 2) respnating at frequencies $f_{1}$ and $f_{2}$, based or (5) we can yrite:

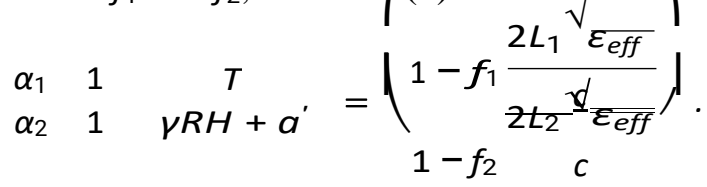

The length $L$ used in (6) is the effective length which takes into account the physical length $I$ of the resonator and the length $\Delta /$ due to fringing fields $(L=I+\Delta /)$. Notice also that $L$ is independent of temperature and humidity. If the criterion $\alpha_{2} \quad \alpha_{1}$ is respected, (6) can be re-written as:

$$
\begin{aligned}
& T=2 V_{\overline{\varepsilon f f}} \frac{f_{2}\left(I_{2}+\Delta l_{2}\right)-f_{1}\left(I_{1}+\Delta I_{1}\right)}{c\left(\alpha_{1}-\alpha_{2}\right)} \\
& L_{R H}=2^{\sqrt{ } \frac{\alpha_{1} f_{2}\left(I_{2}+\Delta / I_{2}\right)-\alpha_{2} f_{1}\left(I_{1}+\Delta I_{1}\right)}{\varepsilon_{e f f}}}-a^{\prime}
\end{aligned}
$$

By measuring the resonance frequencies during a calibration step at two known temperatures, the quantities $\Delta / 1$ and $\Delta / 2$ can be found by solving (7) for $T$. Later, considering all the coefficient as known values [6], by measuring the resonance frequencies of both resonators, we can use (7) to extract the temperature $T$ and the relative humidity $R H$ observed by the resonators. 


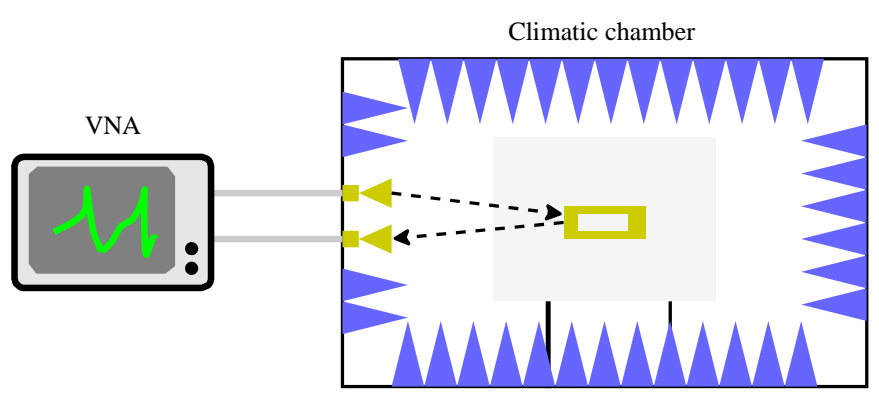

Fig. 1. Principle of the measurement of the scatterer's resonance frequency used to sense temperature and humidity.

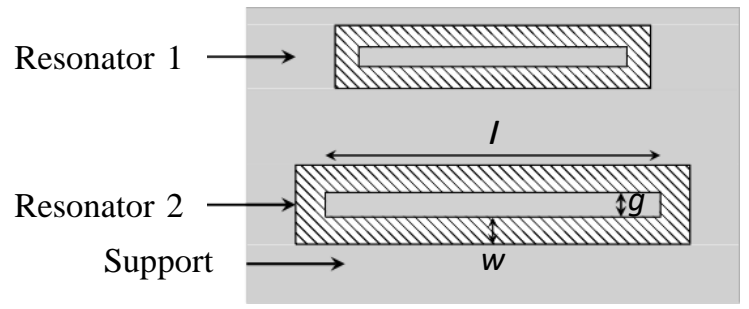

Fig. 2. Schematic of a sensing tag composed of two metal loop on a substrate $\varepsilon_{r}$ scatterers

In order to increase the temperature and humidity accuracy and sensibility, dielectrics with higher temperature and humidity dependencies (like Kapton [8]) as well as a resonator made of a metal with a higher dilatation coefficient (like zinc) should be used. By doing so, coefficients $\alpha_{1}$ and $\alpha_{2}$ in (5) will increased, resulting in higher frequency shifts which are easier to measure. Also by looking at (7), one can notice that the temperature and humidity resolution are immediately linked to the frequency steps used for the measurements. Hence having a lower frequency step increases the resolution of the measurands.

\section{Simulations}

Beforehand, simulations have been carried out to validate the extraction given by (7). The tag composed of two loop scatterers is illustrated in Fig 2. Zinc and copper metals, with respective thermal dilatations $\alpha^{\text {copper }}=17 \times 10^{-6}{ }^{\circ} \mathrm{C}^{-1}$ and $\alpha_{c}^{z i n c}=31 \times 10^{-6}{ }^{\circ} \mathrm{C}^{-1}$ are considered for simulation. The first resonator in copper has $g=2.07 \mathrm{~mm}, w=1.43 \mathrm{~mm}$ and $I=47.97 \mathrm{~mm}$. The second resonator in zinc has the same values for $g$ and $w$ but a different length $I=38.38 \mathrm{~mm}$. The simulated support's permittivity is $\varepsilon_{r}(T, R H)=3.55(1+40 \times$ $\left.10^{-6} R H\right)\left(1+10 \times 10^{-6} T\right)$ where $T$ and $R H$ will vary in a specific range: $0<T<60{ }^{\circ} \mathrm{C}, 0<R H<100 \%$. CST Time Solver is considered using a plane wave excitation. Part of the simulated RCS curves are plotted in Fig. 3. Equation (7) is then applied on the extracted resonance frequencies : the one for resonator 1 is around $2.36 \mathrm{GHz}$, and $2.91 \mathrm{GHz}$ for resonator 2 .

In Fig. 4, it can be seen that the extracted physical quantities does not depend on each other, so that the isolation of the two quantities is verified. The axes in Fig. 4 correspond

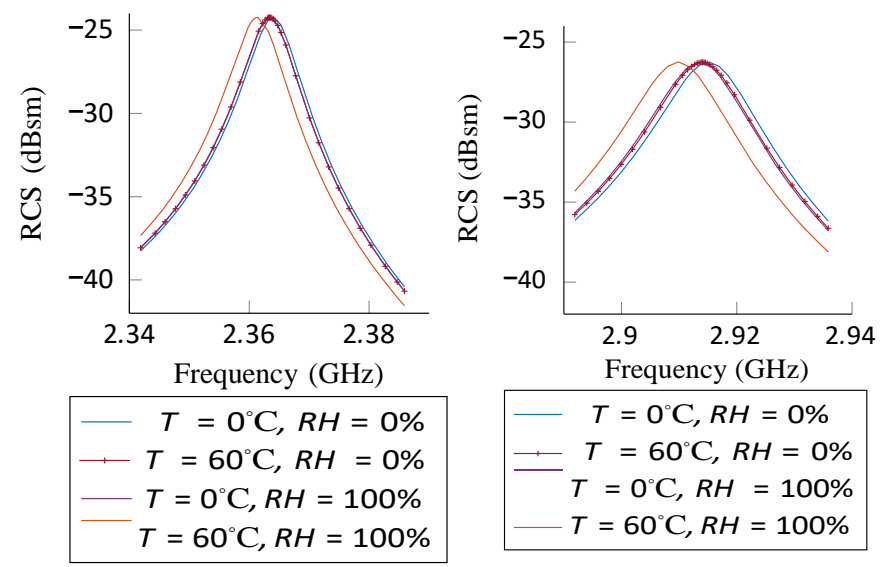

Fig. 3. Simulated RCS response of a sensing tag composed of two scatterers for different temperature and humidity.
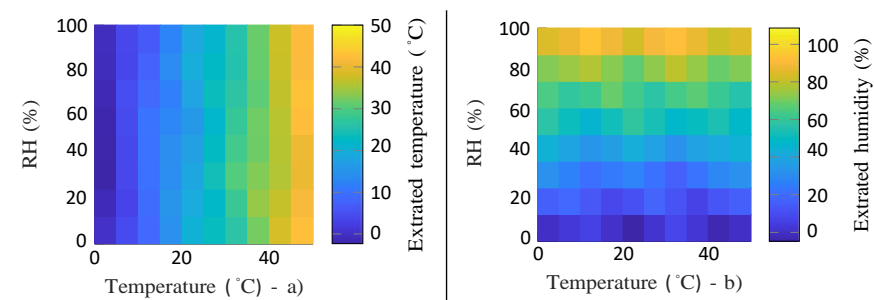

Fig. 4. a) Extracted temperature and b) extracted relative humidity using (7). The simulated chipless tag is shown in Fig. 2. The permittivity of the support is $\varepsilon_{r}=3\left(1+40 \times 10^{-6} R H\right)\left(1+10 \times 10^{-6} T\right)$.

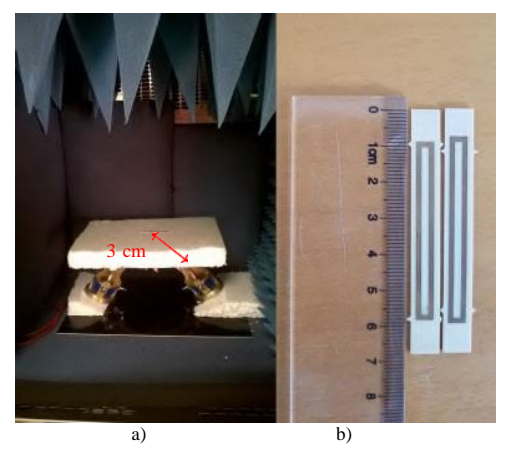

Fig. 5. Photo of the measurement bench and tags. a) Setup inside the climatic chamber. b) The loop resonators used for the measurement. The first resonator has $g=2 \mathrm{~mm}, w=1 \mathrm{~mm}$ and $I=49 \mathrm{~mm}$. The second resonator has the same $\mathrm{g}$ and $\mathrm{w}$ but $I=46 \mathrm{~mm}$.

to the temperature and humidity values imposed in the simulation. The extracted temperature in Fig. 4-a is in good agreement with the simulated temperature with a maximum error of $6^{\circ} \mathrm{C}$ and $2^{\circ} \mathrm{C}$ on average. Fig. 4-b shows the extracted humidity. We can see a good agreement but errors are higher. We can observe a maximum error of $9 \% \mathrm{RH}$ and $3 \% \mathrm{RH}$ on average.

\section{MEASUREMENTS}

The measurement setup and the fabricated tags used in practice are shown in Fig. 5.

A climatic chamber VC0018 by Votsch is used to control temperature and humidity. Absorbers are placed inside the 

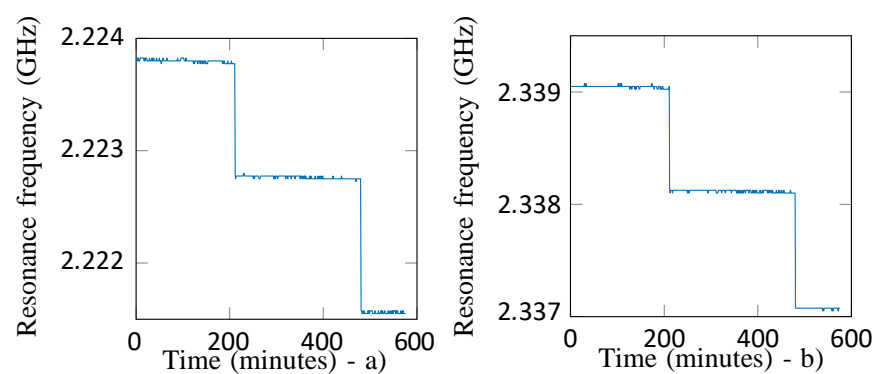

Fig. 6. a) Measurement of the resonance frequency of the first resonator as a function of time. b) Measurement of the resonance frequency of the second resonator as a function of time.

chamber to reduce the reflection level (see Fig. 5-a). A bi-static antenna configuration is used to increase the isolation. For the measurements, the following protocol is used : the temperature in the climatic chamber is first set. When the temperature is stabilized, the $S_{21}$ parameter is measured using a VNA. Temperature and humidity inside the chamber are measured using an electronic sensor. The measured S-parameters have been smoothed in order to remove any residual measurement noise that may impact the search of the resonance frequency and thus induce an error on the frequency extraction.

\section{RESULTS AND ANALYSIS}

A conventional PCB chipless tag was used in practice. The tag is composed of two copper resonators (see Fig. 5-b) on a Rogers RO4003C substrate. The criterion $\alpha_{1} \alpha_{2}$ is respected by the use of different lengths of resonator (see [eq (5), [9]]). The effective permittivity has been calculated using [7] and a value of 1.74 has been obtained. The coefficients $\alpha_{1}=29 \times 10^{-6}{ }^{\circ} \mathrm{C}^{-1}$ and $\alpha_{2}=30 \times 10^{-6}{ }^{\circ} \mathrm{C}^{-1}$ have been calculated using [6]. For this substrate, the humidity variation is not indicated by the provider. For this reason, we have first extracted the value of $y$ and $a^{\prime}$. A first measurement at constant temperature of $20{ }^{\circ} \mathrm{C}$ and a humidity variation from $59 \% \mathrm{RH}$ to $60.5 \% \mathrm{RH}$ is done. Then, (7b) can be use to extract $\gamma$ and $a^{\prime}$. A fitting is done according to the measurement results obtained and (7b). Values of $3.05 \times 10^{-4} \% \mathrm{RH}^{-1}$ and 0.25 are respectively obtained for $\gamma$ and $a^{\prime}$. These coefficients are only valid for a small variation of the humidity around $60 \% \mathrm{RH}$.

Afterwards, a new measurement is done with a variation of both temperature and humidity and the measured resonances frequency are plotted in Fig. 6. Variations of $20{ }^{\circ} \mathrm{C}$ and $6 \% \mathrm{RH}$ are achieved and (7) is applied. The results of the extraction are shown in Fig. 7 alongside with the temperature and humidity measured by the electronic sensor. The highest difference with the electronic temperature sensor is of $3{ }^{\circ} \mathrm{C}$ but these differences are very localized and due to a too high discretization on the measured frequency. Increasing the number of frequency points during the measurement would reduced this error. On average, the differences are lower than $0.7^{\circ} \mathrm{C}$. For the humidity, the previously measured $\gamma$ and $a^{\prime}$ are used. The maximum error done in the humidity measurement is $1.3 \% \mathrm{RH}$.
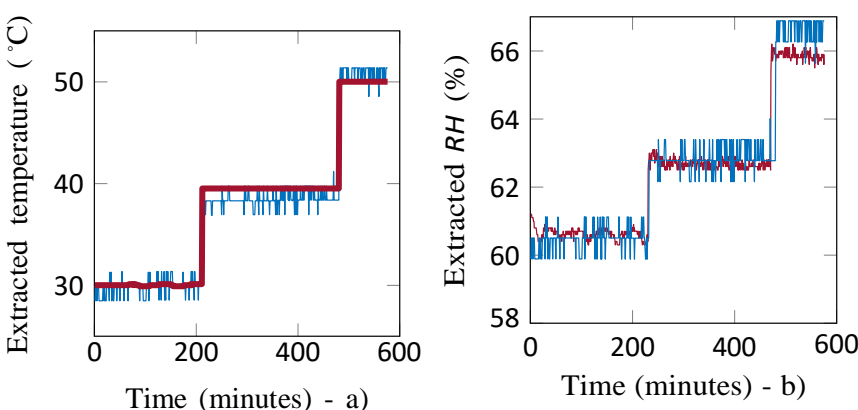

Fig. 7. a) Extracted temperature in blue using (7) and measured temperature with the electronic sensor in red. b) Extracted relative humidity using (7) in blue and measured with the electronic sensor in red. The test-bench is shown is Fig. 5.

Unlike temperature which can often be considered linear for classical materials used for tag fabrication in a range that could be around $0-100^{\circ} \mathrm{C}$, the linearization of humidity in (3) is only valid for a small variation in humidity around a specific value. However, to increase this range of definition, it is possible to use a model with more parameters [compared to (3)] describing a non-linear variation of the permittivity with humidity. This model can be based on a polynomial of order 2 or 3 for example.

\section{CONCLUSION}

This paper introduces a temperature and humidity sensor for RFID chipless applications. This can be used with no additional cost to existing frequency chipless RFID tags while keeping their identification functionality untouched. The model of the sensor is studied in simulation and real-life measurements. Possible applications can be found in food supply, maintenance or even health monitoring.

\section{ACKNOWLEDGMENT}

This project has received funding from the European Research Council (ERC) under the European Union's Horizon 2020 research and innovation program (ScattererID, grant agreement No 772539).

\section{REFERENCES}

[1] R. M. Ferdous, A. W. Reza, and M. F. Siddiqui, "Renewable energy harvesting for wireless sensors using passive RFID tag technology: A review," Renewable and Sustainable Energy Reviews, vol. 58, pp. 1114-1128, 2016.

[2] J. Zhou and J. Shi, "RFID localization algorithms and applications-a review," Journal of intelligent manufacturing, vol. 20, no. 6, p. 695, 2009.

[3] Z. Meng and Z. Li, "RFID tag as a sensor-a review on the innovative designs and applications," Measurement science review, vol. 16, no. 6, pp. 305-315, 2016.

[4] E. M. Amin and N. Karmakar, "Development of a chipless RFID temperature sensor using cascaded spiral resonators," in SENSORS, 2011 IEEE. IEEE, 2011, pp. 554-557.

[5] J. Virtanen, L. Ukkonen, T. Björninen, L. Sydänheimo, and A. Z. Elsherbeni, "Temperature sensor tag for passive UHF RFID systems," in 2011 IEEE Sensors Applications Symposium. IEEE, 2011, pp. 312-317.

[6] R. Florian, G. Michael, B. Nicolas, K. Darine, S. Romain, C. Filippo, G. Simone, and P. Etienne, "Thermal modeling of resonant scatterers and reflectometry approach for remote temperature sensing," submitted to IEEE transactions on microwave theory and techniques, . 
[7] E. Chen and S. Y. Chou, "Characteristics of coplanar transmission lines on multilayer substrates: Modeling and experiments," IEEE transactions on microwave theory and techniques, vol. 45, no. 6, pp. 939-945, 1997.

[8] dupont.com. (2021). [accessed 01 mar. 2021]. [Online]. Available: https://www.dupont.com/content/dam/dupont/amer/us/en/products/ ei-transformation/documents/EI-10142-Kapton-Summary-of-Properties. pdff

[9] F. Requena, N. Barbot, D. Kaddour, and E. Perret, "Contactless characterization of metals thermal expansion coefficient by a free-space RF measurement," IEEE Transactions on Antennas and Propagation, 2020. 